\title{
SPS2
}

\section{Astronomy in Antarctica}

Chairperson: M. Burton

Editor: M. Burton 


\title{
The Potential for Astronomy in Antarctica
}

\author{
M. G. Burton \\ School of Physics, University of New South Wales, Sydney, NSW 2052, \\ Australia
}

\begin{abstract}
The motivation for holding Special Session 2 at the $25^{\text {th }}$ IAU GA is described, together with an outline of the rational for pursuing astronomy in Antarctica.
\end{abstract}

The IAU General Assembly has provided a focal point for discussions on progress of Antarctic astronomy. In the 1991 GA in Buenos Aires a working group on the subject was established, and a resolution passed encouraging its development. At the 1994 GA in Den Haag, CARA, the US Center for Astrophysical Research in Antarctica, had just established itself at the South Pole, and talks focused on the potential of that site. By the 1997 GA in Kyoto, several facilities were in operation and their first results were reported. There was no specific Antarctic Astronomy meeting during the 2000 GA, but by time of the Sydney GA in 2003 several ice-breaking results had been achieved, particularly in measurements of the CMBR and with regards to neutrino detection, together with front-page coverage in journals as esteemed as Nature and the New York Times. Moreover, a new base at the high plateau site of Dome $\mathrm{C}$ was nearing completion, Concordia Station, with the first indications of the site's quite superb quality. The $25^{\text {th }}$ IAU GA therefore saw the opportunity for an more indepth discussion on the opportunities offered by Antarctica. Special Session 2 of the GA on the $19^{\text {th }}$ of July was a meeting to discuss the current status of Antarctic astronomy. The next day at Taronga Zoo, overlooking Sydney Harbor, was held a meeting on 'Visions for Antarctic astronomy' - a blue-sky, free-wheeling discussion limited only by the imagination on what might be possible in Antarctica. In addition, a live video-link up with the astronomers at the South Pole station was held during the lunch break of SPS2, only slightly marred by the sporadic video signal from Pole. However the audio signal came in well, as did the video to Pole, so the Polies saw us and spoke to a power point presentation they had prepared. A fascinating exchange ensued. The papers for these two meetings are presented in this issue of Highlights of Astronomy. The full programs can be found on the meeting web site, www.phys.unsw.edu.au/sps2, which also includes many of the presentations.

For several years it has been clear that the high, dry and cold conditions of the Antarctic plateau provided superb conditions for a wide range of astronomy from infrared to microwave wavelengths. Precipitable water vapor columns falling below $250 \mu \mathrm{m} \mathrm{H}_{2} \mathrm{O}$ open new windows in the IR and sub-mm. Temperatures falling as low as $-80^{\circ} \mathrm{C}$ reduce sky backgrounds by factors of 10-100 times between 2.3 and $30 \mu \mathrm{m}$. Less well known has been the low level of aerosols across the continent, reducing sky emissivities in the IR. The high geomagnetic latitudes has been put to good use for many years for cosmic ray detection through 
the lower energy particles accessible. The vast quantities of pure ice has been employed to build downward pointing neutrino detectors, with large collecting volumes and minimal levels of background contamination.

A decades experience of operating complex facilities at the South Pole has shown that operation on the plateau was quite feasible. It was readily accessible by aircraft. Conditions were generally calm, and there were never any gales. Working wasn't actually that hard, once one had adequately prepared for the conditions and designed experiments appropriately. And there were no Polar Bears or other distractions to worry about!

Two other facets of the Antarctic plateau were, however, only just becoming apparent by the time of the Sydney GA. The first was the incredible stability of the air column above the summits of the plateau. As the IAU GA was taking place, the first season of winter time measurements from Dome $\mathrm{C}$ was also occurring, made from the completely autonomous AASTINO laboratory. Thanks to the Iridium satellite communications system their results were available at the GA, suggesting that the skies were clear for nearly all the time and that the micro-turbulence in the boundary layer was much less than at South Pole. While further data is still required of the entire air column, the indications are that the isoplanatic angle will be well over an order of magnitude greater than at temperate latitude sites, and scintillation noise correspondingly reduced. The conditions thus appear extraordinarily favorable for adaptive optics correction. The second 'new' facet about the plateau was that it was tectonically stable. While this had in fact been known previously, the implications this has for the construction of the next generation of extremely large optical telescopes were only just becoming apparent.

With the quantification of the site qualities of the Antarctic plateau becoming pinned down, clear areas where the conditions provide particular niches for observational astronomy have emerged, for instance:

- The imaging of cosmic sources of neutrino emission.

- Precision measurements of the cosmic microwave background, including its power spectrum, polarization and the SZ-effect.

- Wide-field thermal infrared imaging, from 3 to $30 \mu \mathrm{m}$, to uncover all sites of star formation across the Galaxy and the LMC.

- Continuous observation of objects, particularly in the dark 'cosmological' window at $2.4 \mu \mathrm{m}$.

- Precision photometry, for instance for measurement of planetary transits of stars or stellar seismology.

- Mid-infrared photometry, for instance applied to the detection of planetary systems.

- Astrometric interferometry, with micro-arcsecond precision, with the capability of measuring three dimensional motions across the Galaxy.

In the following pages are papers on Antarctic astronomy which outline many of these results and developments. 